\title{
A Randomised, Controlled Study of Different Glycaemic Targets during Gestational Diabetes Treatment: Effect on the Level of Adipokines in Cord Blood and ANGPTL4 Expression in Human Umbilical Vein Endothelial Cells
}

\author{
P. Popova $\mathbb{D}^{1},{ }^{1,2}$ L. Vasilyeva, ${ }^{1}$ A. Tkachuck, ${ }^{1}$ M. Puzanov, ${ }^{1}$ A. Golovkin ${ }^{1 D},{ }^{1}$ Y. Bolotko, ${ }^{1}$ \\ E. Pustozerov, ${ }^{1,3}$ E. Vasilyeva, ${ }^{1}$ O. Li, ${ }^{1}$ I. Zazerskaya, ${ }^{1}$ R. Dmitrieva, ${ }^{1}$ A. Kostareva, ${ }^{1}$ \\ and E. Grineva $\mathbb{D}^{1,2}$ \\ ${ }^{1}$ Almazov National Medical Research Centre, Saint Petersburg, Russia \\ ${ }^{2}$ Department of Internal Diseases and Endocrinology, St. Petersburg Pavlov State Medical University, Saint Petersburg, Russia \\ ${ }^{3}$ Department of Biomedical Engineering, Saint Petersburg State Electrotechnical University, Saint Petersburg, Russia
}

Correspondence should be addressed to P. Popova; pvpopova@yandex.ru

Received 4 January 2018; Revised 27 February 2018; Accepted 14 March 2018; Published 14 May 2018

Academic Editor: Agnieszka Rak

Copyright (c) 2018 P. Popova et al. This is an open access article distributed under the Creative Commons Attribution License, which permits unrestricted use, distribution, and reproduction in any medium, provided the original work is properly cited.

Our aim was to study the expression of adipokine-encoding genes (leptin, adiponectin, and angiopoietin-like protein 4 (ANGPTL4)) in human umbilical vein endothelial cells (HUVECs) and adipokine concentration in cord blood from women with gestational diabetes mellitus (GDM) depending on glycaemic targets. GDM patients were randomised to 2 groups per target glycaemic levels: GDM1 (tight glycaemic targets, fasting blood glucose $<5.1 \mathrm{mmol} / \mathrm{L}$ and $<7.0 \mathrm{mmol} / \mathrm{L}$ postprandial, $N=20$ ) and GDM2 (less tight glycaemic targets, $<5.3 \mathrm{mmol} / \mathrm{L}$ and $<7.8 \mathrm{mmol} / \mathrm{L}$, respectively, $N=21$ ). The control group included 25 women with normal glucose tolerance. ANGPTL4 expression was decreased in the HUVECs from GDM patients versus the control group $(23.11 \pm 5.71,21.47 \pm 5.64$, and $98.33 \pm 20.92$, for GDM1, GDM2, and controls; $p<0.001)$ with no difference between GDM1 and GDM2. The level of adiponectin gene expression was low and did not differ among the groups. Leptin gene expression was undetectable in HUVECs. In cord blood, leptin/adiponectin ratio (LAR) was increased in GDM2 compared to controls and GDM1 $(p=0.038)$ and did not differ between GDM1 and controls. Tight glycaemic targets were associated with normalisation of increased LAR in the cord blood. ANGPTL4 expression was downregulated in HUVECs of newborns from GDM mothers and was not affected by the intensity of glycaemic control.

\section{Introduction}

The intrauterine hyperglycaemia in women with gestational diabetes mellitus (GDM) is supposed to be an important factor that predisposes offspring to obesity and type 2 diabetes mellitus (T2D) $[1,2]$. However, the mechanisms connecting intrauterine exposure to hyperglycaemia with subsequent development of metabolic diseases are not clear enough.

There is some suggestion that the exposure to diabetes in utero increases the risk of offspring obesity via alterations in the "adipoinsular axis," the endocrine loop, linking the brain and endocrine pancreas with insulin- and leptinsensitive tissues in the control of eating behaviour and energy balance $[1,3]$.

Adipokines play an important role in the energy metabolism regulation [4]. Leptin (LEP) and adiponectin (ADIPOQ) are well-recognised obesity- and diabetes-related candidate genes through which the adipose tissue influences the regulation of several important physiological functions, including appetite, satiety, energy expenditure, insulin sensitivity, fat 
distribution, and endothelial function [4]. Adiponectin and leptin are also factors associated with fetal growth [5] and shown as predictors of early-life weight gain $[6,7]$.

Another promising adipokine is angiopoietin-like protein 4 (ANGPTL4), a multifunctional signal protein expressed in many tissues. ANGPTL4 is involved in the regulation of multiple physiological processes, including energy metabolism [8], plasma glucose level and tolerance regulation [9], fat storage, and lipid metabolism [10]. The association of ANGPTL4 expression with obesity was confirmed in a study of monozygotic twins [11]. Robciuc et al. revealed that ANGPTL4 expression in the adipose tissue and circulation was inversely correlated to body weight, suggesting a role for ANGPTL4 in acquired obesity [11].

The change in the expression of the abovementioned genes in the fetal tissues may serve as a marker of subsequent metabolic diseases of the offspring. The association between the presence of hyperglycaemia in the mother and altered cord blood levels of leptin, adiponectin, and ANGPTL4 has been identified in previous studies $[5,12,13]$. An increased placental LEP expression level has been also described in women with GDM [14].

However, it is not obvious that maternal hyperglycaemia causes such alterations. Perhaps, on the contrary, the altered gene expression functions in GDM pathogenesis (e.g., due to activation of hormone-encoding genes evoking insulin resistance or reduction of insulin secretion). It is also possible that both phenomena (maternal hyperglycaemia and changes in the expression of adipokines in the fetus and/or the mother) result from other pathological processes.

Randomised controlled trials (RCT) comparing changes of newborn gene expression level in groups of women with different target glucose levels during the treatment of GDM are supposed to help clarifying the cause-and-effect relations.

The human umbilical vein endothelial cells (HUVECs) represent a good cellular model for studying the effect of maternal hyperglycaemia on the fetal cardiovascular system and can serve as a marker of the predisposition of the fetus to metabolic diseases [15].

In this study, we investigated the alterations in ANGPT4, ADIPOQ, LEP, and leptin receptor gene (LEPR) expression levels in HUVECs and concentrations of these adipokines in the cord blood from newborns of women with GDM with different glycaemic targets compared to healthy women.

\section{Materials and Methods}

This study was carried out at the Almazov National Medical Research Centre (ANMRC) as part of the ongoing RCT "Genetic and epigenetic mechanisms of developing gestational diabetes mellitus and its effects on the fetus" (GEM GDM) which started in July 2015. This study was approved by the local ethical committee (Protocol 119); informed written consent was obtained from all subjects.

2.1. Design and Study Population. Forty-one women with GDM and 25 controls were randomly selected to assess the levels of expression of genes in HUVECs. The women with GDM were randomised to 2 groups according to target glycaemic levels: group 1 (target fasting blood glucose $<5.1 \mathrm{mmol} / \mathrm{L}$ and $<7.0 \mathrm{mmol} / \mathrm{L}$ 1-hour postprandial) (GDM1, $N=21$ ) and group 2 (target fasting blood glucose $<5.3 \mathrm{mmol} / \mathrm{L}$ and $<7.8 \mathrm{mmol} / \mathrm{L}$ 1-hour postprandial) (GDM2, $N=20$ ).

GDM was diagnosed according to the Russian National Consensus [16] and the recommendations of the International Association of Diabetes and Pregnancy Study Groups (IADPSG) based on the results of 2-hour oral glucose tolerance test (OGTT) performed at 24th-28th week of gestation [17]. Pregnant women without diabetes were included as controls.

None of the patients had previous history of diabetes mellitus or any known medical condition affecting glucose metabolism.

They were all followed until delivery at ANMRC. Anthropometric variables (height and blood pressure) were measured using standardised procedures. Prepregnancy body mass index (BMI) was calculated based on the prepregnancy weight recalled by participants. Women with GDM were consulted by the endocrinologist and provided the results of their self-measurements of blood glucose every 23 weeks. In case of exceeding the target blood glucose levels (in 2 or more measurements per week in group 1 and in more than $1 / 3$ of measurements per week in group 2), insulin therapy was started. The participants were asked to keep electronic nutrition and glycaemic control diaries with the help of a specially developed mobile application and send data to the doctor. The mobile application is described elsewhere [18]. According to the personal diaries, automatic calculations of the integral indicators characterising the selfcontrol of glycaemia (fasting, postprandial, and average glycaemia) were accomplished. Electronic diary data were available for almost all women with GDM $(N$ GDM1 $=19, N$ GDM2 $=20)$ and 8 women from the control group.

2.2. Blood Sample Processing and Analysis. Cord blood samples were collected immediately after delivery. Blood glucose measurements were made on fresh plasma samples. The cord blood serum samples were stored at $-80^{\circ} \mathrm{C}$ for further analysis of C-peptide, leptin, adiponectin, and ANGPTL4. Plasma glucose (PG) concentration was determined by the glucose oxidase method. Serum C-peptide level was measured by the chemiluminescent microparticle immunoassay (Architect C-peptide assay, Abbott Laboratories, IL, USA). Serum adiponectin (BioVendor Laboratory Medicine Inc., Modrice, Czech Republic) and leptin (Diagnostics Biochem Canada Inc., Canada) levels were measured using an enzyme-linked immunosorbent assay (ELISA) as recommended by the manufacturer. Serum level of ANGPTL4 was determined by DuoSet ELISA Development kits (DY3485) from R\&D Systems (USA). The limit of detection for ANGPTL4 is $1.25 \mathrm{ng} / \mathrm{mL}$. The detection range is $1.25 \mathrm{ng} / \mathrm{mL}-80 \mathrm{ng} / \mathrm{mL}$. The following factors prepared at $800 \mathrm{ng} / \mathrm{mL}$ were assayed and exhibited no cross-reactivity or interference: recombinant human angiopoietin-1, angiopoietin-2, angiopoietin-4, and angiopoietin-like 3 and recombinant mouse angiopoietin-3 and angiopoietin-like 3. 
The limit of detection for Leptin is $0.5 \mathrm{ng} / \mathrm{mL}$. The detection range is $0.5-100 \mathrm{ng} / \mathrm{mL}$.

The following substances were tested at $1000 \mathrm{ng} / \mathrm{mL}$ and exhibited no cross-reactivity: mouse leptin, TNF- $a$, IL-2, IL3, IL-4, IL-6, IL-8, IL-9, IL-10, IL-12, IL-16, GM-CSF, CSF, and EGF.

The limit of detection for adiponectin is $26 \mathrm{ng} / \mathrm{mL}$. The detection range is $26 \mathrm{ng} / \mathrm{mL}-100 \mathrm{ug} / \mathrm{mL}$. No cross-reactivity has been observed for human leptin and leptin receptor.

Intra-assay coefficients of variation (CVs) for leptin assay were between $3.7 \%$ and $5.5 \%$, and interassay CVs were $5.8-$ $6.8 \%$. For adiponectin assay, the intra- and interassay CVs were $3.9-5.9 \%$ and $6.3-7.0 \%$, respectively.

2.3. Isolation and Identification of the HUVECs. The HUVECs were isolated using a standard collagenase digestion method [19] as we do routinely in our laboratory [20]. Immediately after isolation, the cells were cultured and expanded in endothelial cell medium (ECM cat number 1001, ScienCell, San Diego, CA) containing 5\% fetal bovine serum, $1 \%$ penicillin/streptomycin, and endothelial cell growth supplement in a humidified atmosphere of 95\% air and $5 \% \mathrm{CO}_{2}$ at $37^{\circ} \mathrm{C}$. For this study, $80 \%$ of confluent HUVEC monolayers (passages 2-3) were used.

The purity of primary HUVEC cultures was evaluated by flow cytometry analysis performed on Guava EasyCyte8. Briefly, detached cells were resuspended in $200 \mu \mathrm{L}$ of PBS containing $1 \%$ bovine serum albumin (Sigma-Aldrich, Saint Luis, MO, USA) and incubated for $15 \mathrm{~min}$ at $20^{\circ} \mathrm{C}$ with the following antibodies (Ab): FITC-conjugated anti-CD31, PE-A-conjugated anti-CD144, PE-Cy7-A-conjugated anti-CD146 (BioLegend, San Diego, CA, USA), PE-A-conjugated anti-CD105 (Bioscience Pharmingen, San Jose, CA, USA), and APC-A-conjugated anti-CD45 (DAKO, Santa Clara, CA, USA). Data files were collected and analysed using the FACSDiva software program (version 6.1.3; BD Bioscience, San Jose, CA, USA).

2.4. Evaluation of Apoptosis and Immunocytochemical Assay. The viability of HUVEC was assessed by flow cytometry with the determination of the number of viable cells, as well as those in early and late apoptosis and necrosis evaluated by Annexin-V/PI (BioLegend, San Diego, CA, USA) double staining.

The expression of von Willebrand factor and CD146 (BioLegend, San Diego, CA, USA) in HUVECs was detected by immunocytochemical staining. Cell nucleuses were stained with $4^{\prime}$,6-diamidino-2-phenylindole (DAPI).

2.5. $R T-q P C R$. Total RNA was extracted from HUVEC using ExtractRNA reagent (BC032, Evrogen, Moscow, Russia) according to the manufacturer's protocol. One microgram of total RNA was reverse transcribed using Moloney Murine Leukemia Virus Reverse Transcriptase (MMLV RT) kit (SK021, Evrogen, Moscow, Russia). After cDNA synthesis, quantitative real-time PCR was performed in $25 \mu \mathrm{L}$ reaction mixture containing: 5x qPCRmix-HS LowROX (PK154L, Evrogen, Moscow, Russia) diluted to a final concentration of $1 \mathrm{x}, 20 \mathrm{x}$ primers diluted to $1 \mathrm{x}, 50 \mathrm{ng} \mathrm{cDNA}$, and deionised distilled water. Reaction mixtures were incubated for an initial denaturation at $95^{\circ} \mathrm{C}$ for $10 \mathrm{~min}$, which was followed by 40 PCR cycles, each consisting of exposure to $95^{\circ} \mathrm{C}$ for $15 \mathrm{sec}$ and $60^{\circ} \mathrm{C}$ for $1 \mathrm{~min}$. Gene expression was evaluated by real-time PCR using Applied Biosystems TaqMan Gene Expression Assays (ADIPOQ: Hs00605917_m1; LEP: Hs00174877_m1; LEPR: Hs00174497_m1; and ANGPTL4: Hs01101127_m1). All data are expressed as ratio to the reference gene GAPDH (forward AATGAAGGGGTCATTG ATGG, reverse AAGGTGAAGGTCGGAGTCAA) (AlkorBio, Saint-Petersburg, Russia).

Relative expression was evaluated according to the $2^{-\Delta \Delta \mathrm{Ct}}$ method [21]. In order to confirm the correctness of the method of detection of LEP and ADIPOQ expression, we used RNA samples derived from our previous adipose differentiation experiments [22]. RNA samples from adipose tissue multipotent mesenchymal stromal cells (MSC) and from differentiated in vitro adipose tissue were used as negative and positive controls, respectively.

2.6. Data Analysis. Statistical analysis was performed using SPSS 22.0 (SPSS Inc., USA). Mean and standard deviation are reported for continuous variables, and numbers and percentages are reported for categorical variables. Differences among the groups were analysed by Mann-Whitney test (for comparison between two groups), Kruskal-Wallis test (for comparison of more than two groups) or chi-square test. A $p$ value $<0.05$ was considered statistically significant.

\section{Results}

3.1. Characteristics of the Participants. Baseline characteristics of the participants are described in Table 1. The women from all three groups did not differ in terms of age and prepregnancy BMI. The GDM1 group had higher diastolic BP compared to controls $(p=0.003)$. The GDM1 and GDM2 groups had higher levels of fasting PG $(p=0.004$ and $p=0.003$, resp.) and higher levels of $P G 1 \mathrm{~h}$ and $2 \mathrm{~h}$ in OGTT $(p<0.001$ in comparison with controls).

Mean levels of fasting, 1-hour postprandial and average blood glucose measured by the participants during the study are described in Table 2. The GDM1 group achieved significantly lower average and 1-hour postprandial glucose levels compared to GDM2. Mean postprandial BG was lower in GDM1 even compared to the control group, though the difference did not reach statistical significance $(p=0.088)$. Gestational weight gain did not differ between GDM1 and GDM2 groups and was lower in both groups compared to controls (Table 2). The percentage of women treated with insulin was $40 \%$ and $29 \%$ in the GDM1 and GDM2 groups, respectively, and did not significantly differ $(p=0.495)$.

3.2. Pregnancy Outcomes. Pregnancy outcomes and biochemical markers in cord blood are shown in Table 3.

There was no statistically significant difference among the groups in terms of pregnancy outcomes (percent of large for gestational age (LGA) and small for gestational age (SGA) newborns, delivery by caesarean section) and the level of Cpeptide, adiponectin, and ANGPTL4 in cord blood serum 
TABLE 1: Characteristics of the participants at study entry.

\begin{tabular}{|c|c|c|c|c|c|c|c|}
\hline & $\begin{array}{c}\text { GDM } 1 \\
(N=20)\end{array}$ & $\begin{array}{c}\text { GDM2 } \\
(N=21)\end{array}$ & $\begin{array}{l}\text { Control } \\
(N=25)\end{array}$ & $p$ & $p$ control-GDM1 & $p$ control-GDM2 & $p$ GDM1-GDM2 \\
\hline Maternal age, years & $30.9 \pm 5.4$ & $32.3 \pm 5.0$ & $30.8 \pm 4.2$ & 0.566 & & & \\
\hline Prepregnancy BMI, kg/m² & $25.4 \pm 7.2$ & $26.1 \pm 6.5$ & $23.4 \pm 4.2$ & 0.287 & & & \\
\hline BP syst, $\mathrm{mmHg}$ & $120 \pm 13$ & $118 \pm 12$ & $112 \pm 14$ & 0.114 & & & \\
\hline $\mathrm{BP}$ diast, $\mathrm{mmHg}$ & $76 \pm 8$ & $73 \pm 10$ & $69 \pm 8$ & 0.016 & 0.003 & 0.155 & 0.195 \\
\hline Fasting PG, mmol/L & $5.1 \pm 0.8$ & $5.0 \pm 0.6$ & $4.5 \pm 0.4$ & 0.007 & 0.004 & 0.003 & 0.396 \\
\hline OGTT $1 \mathrm{~h} \mathrm{PG}, \mathrm{mmol} / \mathrm{L}$ & $10.2 \pm 1.4$ & $9.9 \pm 1.6$ & $6.9 \pm 1.9$ & $<0.001$ & $<0.001$ & $<0.001$ & 0.454 \\
\hline OGTT $2 \mathrm{~h}$ PG, mmol/L & $8.0 \pm 1.6$ & $8.8 \pm 1.6$ & $5.9 \pm 1.5$ & $<0.001$ & $<0.001$ & $<0.001$ & 0.231 \\
\hline Fasting leptin, ng/mL & $22.2 \pm 20.7$ & $29.5 \pm 26.2$ & $26.6 \pm 17.0$ & 0.561 & & & \\
\hline Fasting adiponectin, $\mathrm{ng} / \mathrm{mL}$ & $7.2 \pm 3.3$ & $9.1 \pm 3.3$ & $8.9 \pm 2.5$ & 0.077 & & & \\
\hline
\end{tabular}

Note: BMI: body mass index; BP: blood pressure; PG: plasma glucose; OGTT: oral glucose tolerance test.

TABLE 2: Blood glucose data from electronic diaries and gestational weight gain.

\begin{tabular}{lccccccc}
\hline & $\begin{array}{c}\text { GDM 1 } \\
(N=20)\end{array}$ & $\begin{array}{c}\text { GDM2 } \\
(N=21)\end{array}$ & $\begin{array}{c}\text { Control } \\
(N=25)\end{array}$ & $p$ & $p$ control-GDM1 & $p$ control-GDM2 & $p$ GDM1-GDM2 \\
\hline Gestational weight gain, kg & $9.9 \pm 4.9$ & $9.5 \pm 5.9$ & $15.2 \pm 7.8$ & 0.006 & 0.023 & 0.023 & 0.970 \\
BG average, mmol/L* & $5.6 \pm 0.3$ & $5.9 \pm 0.4$ & $6.0 \pm 0.5$ & 0.004 & 0.110 & 0.893 & 0.421 \\
Fasting BG, mmol/L* & $4.7 \pm 0.4$ & $4.8 \pm 0.3$ & $4.7 \pm 0.3$ & 0.499 & 0.835 & 0.818 & 0.735 \\
1 h postprandial BG, mmol/L* & $5.9 \pm 0.3$ & $6.4 \pm 0.5$ & $6.5 \pm 0.7$ & 0.002 & 0.088 & $<0.001$ & 0.946 \\
Number of BG measurements & $140 \pm 78$ & $147 \pm 60$ & $42 \pm 21$ & 0.001 & $<0.001$ & & \\
\% $(N)$ treated with insulin & $40 \%(8)$ & $29 \%(6)$ & N/A & 0.495 & & & \\
\hline
\end{tabular}

Note: * derived from electronic diaries filled in by participants $(N$ GDM1 $=19, N$ GDM2 $=20, N$ control = 8) during the study period. BG: blood glucose; N/A: nonapplicable.

TABLE 3: Pregnancy outcomes, biochemical markers in the cord blood, and ANGPTL4 gene expression in HUVECs.

\begin{tabular}{|c|c|c|c|c|}
\hline & $\begin{array}{l}\text { GDM } 1 \\
(N=20)\end{array}$ & $\begin{array}{c}\text { GDM2 } \\
(N=21)\end{array}$ & $\begin{array}{l}\text { Control } \\
(N=25)\end{array}$ & $p$ \\
\hline Gestational age at delivery, weeks & $39.2 \pm 1.5$ & $39.3 \pm 1.0$ & $39.7 \pm 1.0$ & 0.261 \\
\hline Caesarean section, $\%(N)$ & $30 \%(6)$ & $19 \%(4)$ & $20 \%(5)$ & 0.723 \\
\hline Birth weight, g & $3572 \pm 488$ & $3584 \pm 577$ & $3513 \pm 555$ & 0.856 \\
\hline Height, cm & $52.1 \pm 2.5$ & $52.4 \pm 2.3$ & $52.1 \pm 2.5$ & 0.990 \\
\hline LGA, \% $(N)$ & $20 \%(4)$ & $23 \%(5)$ & $12 \%(3)$ & 0.235 \\
\hline SGA, \% $(N)$ & $5 \%(1)$ & $9.5 \%(2)$ & $4 \%(1)$ & 0.819 \\
\hline Apgar score $1 \mathrm{~min}$ & $7.5 \pm 0.7$ & $7.7 \pm 1.1$ & $7.7 \pm 0.6$ & 0.204 \\
\hline Apgar score $5 \mathrm{~min}$ & $8.6 \pm 0.5$ & $8.7 \pm 0.9$ & $8.8 \pm 0.4$ & 0.208 \\
\hline Glucose, mmol/L & $4.7 \pm 1.2$ & $5.3 \pm 1.3$ & $4.5 \pm 1.2$ & 0.203 \\
\hline C-peptide, ng/mL & $0.8 \pm 0.5$ & $1.0 \pm 0.6$ & $0.9 \pm 0.4$ & 0.379 \\
\hline Leptin, ng/mL & $8.8 \pm 6.6^{\mathrm{a}}$ & $18.3 \pm 16.1$ & $10.6 \pm 10.4$ & 0.042 \\
\hline Adiponectin, ng/mL & $15.9 \pm 11.5$ & $16.3 \pm 14.4$ & $18.3 \pm 14.3$ & 0.843 \\
\hline LAR & $0.97 \pm 1.31$ & $1.70 \pm 1.66^{\mathrm{b}}$ & $0.72 \pm 0.46$ & 0.038 \\
\hline ANGPTL4 in cord serum, $\mathrm{ng} / \mathrm{mL}$ & $19.9 \pm 15.0$ & $14.1 \pm 4.5$ & $13.9 \pm 5.2$ & 0.248 \\
\hline ANGPTL4 relative expression in HUVECs & $23.1 \pm 25.6^{\mathrm{c}}$ & $21.5 \pm 25.8^{\mathrm{c}}$ & $98.3 \pm 104.6$ & 0.001 \\
\hline
\end{tabular}

Notes: LAR: leptin/adiponectin ratio; LGA: large for gestational age; SGA: small for gestational age. LGA was defined by a birth weight exceeding the 90th percentile on standard charts. SGA was defined by a birth weight below the 10th percentile on standard charts. ${ }^{a} p<0.05$ versus GDM2; ${ }^{b} p<0.05$ versus the control group; ${ }^{c} p<0.01$ versus the control group.

and glucose in cord blood plasma. The level of leptin in cord blood serum was higher in the GDM2 group than in the GDM1 $(p=0.036)$ and the control group, but the difference from the control group did not reach statistical significance $(p=0.066)$. After adjustment by insulin therapy, age, and prepregnancy BMI, the difference in the level of leptin 
between GDM1 and GDM2 remained significant ( $p=0.01)$. The leptin/adiponectin ratio (LAR) in cord blood serum was higher in the GDM2 group compared to controls $(p=0.011)$ with no difference between the GDM1 and control group $(p=0.404)$.

3.3. HUVEC Characterisation. HUVECs were obtained from the umbilical vein, expanded in vitro, and characterised for expression of endothelial markers by flow cytometry and immunohistochemistry. All samples demonstrated characteristic endothelial morphology and immunophenotype CD45-/CD144+/CD31+/CD146+/CD105+ and stained positively for endothelial markers, von Willebrand factor, and CD146 (as demonstrated in our previous work [20]). There was no difference in the parameters of viability and replicative aging of HUVEC cultures from different patient groups (data not presented).

3.4. Gene Expression in HUVECs. ANGPTL4 expression was downregulated in the HUVECs derived from GDM patients compared to control group $(23.11 \pm 5.71,21.47 \pm 5.64$, and $98.33 \pm 0.92$, respectively, for GDM1, GDM2, and control groups; $p<0.001$ for comparison among the 3 groups) while no difference between GDM1 and GDM2 groups was observed (Figure 1(a)).

We did not detect the expression of LEP in HUVEC but found out that they expressed LEPR, and the expression of LEPR demonstrated a decline in the GDM1/GDM2 groups compared to the control group, though the differences were not statistically significant (Figure 1(c)). The expression of LEPR did not correlate with the level of LEP in cord plasma (Figure 1(d)) which indicates that there is no reciprocal regulation between LEP and its receptor in HUVECs. The expression of ADIPOQ was detected in HUVEC samples, but the level of its expression was as low as in negative control samples and lower by four orders of magnitude in HUVEC compared to adipocytes (positive control) (Figure 1(b)).

\section{Discussion}

Our RCT has shown that GDM treated according to the most widely accepted current guidelines was associated with the increased LAR in cord blood and that LAR did not differ from the control group if GDM was treated aiming at tighter glycaemic targets. The GDM1 group (with tighter glycaemic targets) had lower levels of leptin compared to GDM2. We also found a decrease in the level of expression of ANGPTL4 in HUVEC of newborns from women with GDM in comparison with the control group. However, there was no difference in the level of expression of ANGPTL4 between the two groups of GDM with different glycaemic targets.

The most appropriate target levels of glycaemia for the management of GDM are not universally defined [23]. Most organisations [24-26] suggest the targets for glycaemic control for women with GDM based on recommendations from the Fifth International Workshop-Conference on Gestational Diabetes Mellitus [27].
These targets were used by the Maternal-Fetal Medicine Units Network (MFMU) trial showing benefit for the treatment of GDM [28]. We used these targets for group 2.

However, there are no reliable data from controlled trials of lower versus higher target levels of glycaemia to identify ideal glycaemic targets for prevention of fetal risks [29].

The glycaemic targets used in our study for group 1 were tighter in accordance with current Russian guidelines [16]. Our data suggest that achieving tighter glycaemic targets during GDM treatment reduces LAR in the cord blood. However, this data should be interpreted with caution considering the small sample size. The full information about pregnancy outcomes is needed to guide clinical practice regarding target glycaemic levels during pregnancy. Our current study with a small sample size was not designed for this purpose. However, our findings may serve as a confirmation of the cause-and-effect relationship between maternal hyperglycaemia and alteration of LAR in newborns. Our results are in line with the data by Pirc et al. that reported treatment of mild GDM reduces cord blood leptin [12]. The authors hypothesise that hyperleptinaemia of the babies born to women with untreated mild GDM may persist for some time. It could reduce appetite and food intake and might contribute to the phenomenon of catch-down growth seen in macrosomic infants following birth $[12,30]$.

Follow-up studies are needed to understand the impact of tight glycaemic targets during pregnancy on obesity development in the offspring of women diagnosed with GDM according to IADPSG criteria. It is especially important taking into consideration the evidence that low earlylife leptin concentrations may promote faster weight gain in infancy [31-33].

There is controversy about the association of adiponectin levels in cord blood with GDM. Pirc et al. reported decreased levels of adiponectin in the cord blood of newborns from mothers with GDM [12], whereas several other studies, like ours, find no effect of maternal DM on cord blood adiponectin concentration [5, 34, 35]. The reasons for these differences are unclear but may reflect different assay methodologies, different study populations, and different criteria used to diagnose GDM.

The level of expression of LEP in HUVECs turned out to be below the detection threshold. The expression of ADIPOQ was detected in HUVEC samples, but the level of its expression was as low as in negative control samples, which confirms that umbilical vein endothelium is not a place of adiponectin production. We are not aware of any other study addressing the expression of these genes in HUVEC. However, our results are in line with some of the previous studies which have shown that ADIPOQ is not expressed in the placenta $[14,36]$. Thus, the levels of cord adiponectin may be attributed to other fetal tissues, while LEP has been shown to be expressed in the placenta [14]. It should be noted that there are also conflicting results of the studies which indicated the expression of ADIPOQ in human placenta [36, 37].

In contrast to LAR changes associated with tight glycaemic targets of treatment of GDM, the level of expression of ANGPTL4 was lower in both the GDM groups regardless of glycaemic targets compared to the control group. Possibly, 


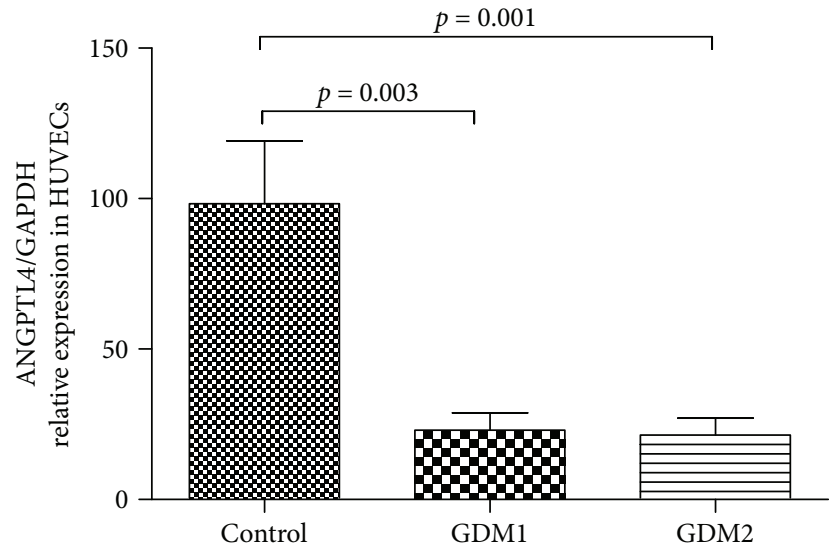

(a)

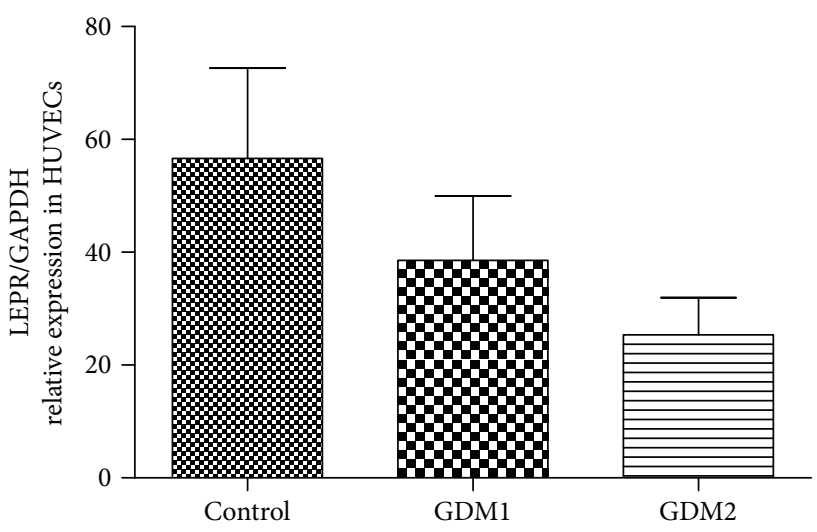

(c)

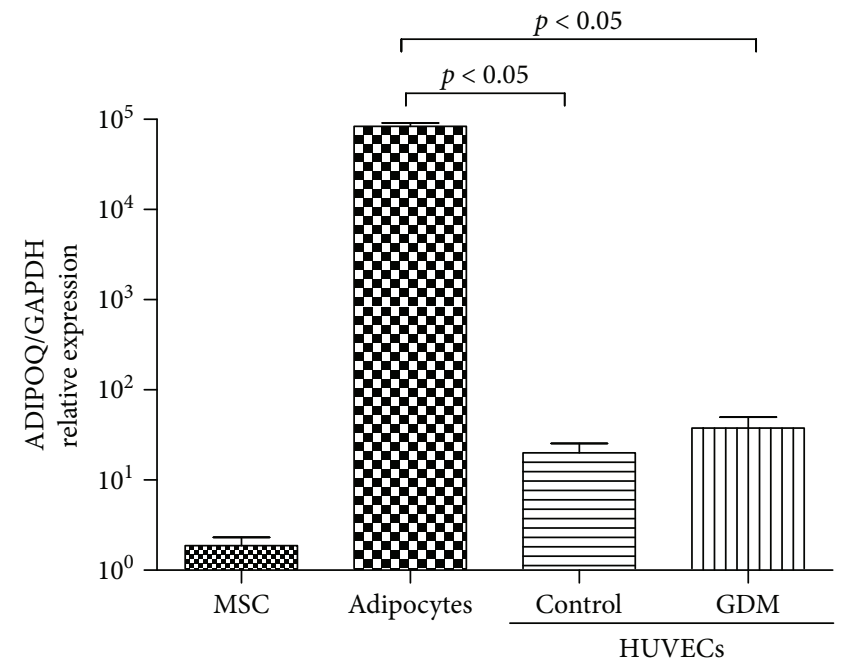

(b)

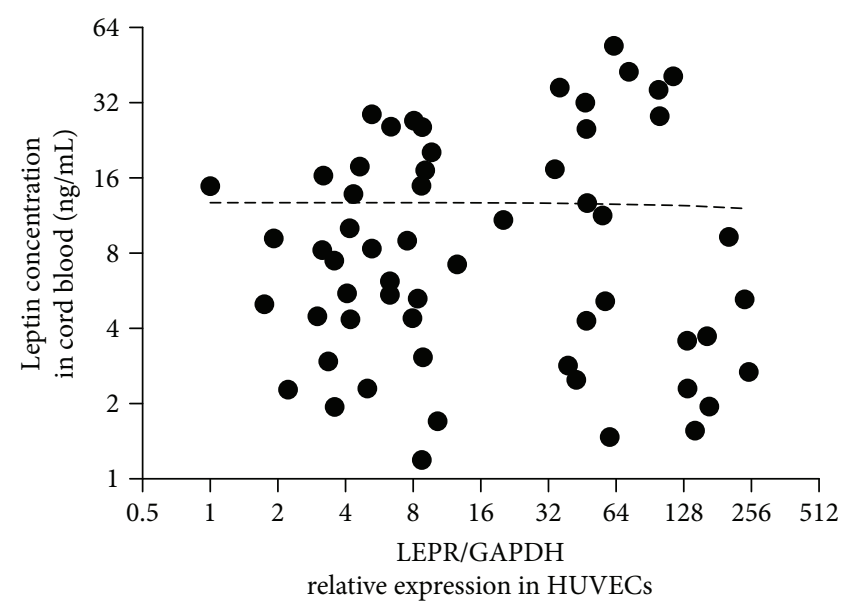

(d)

Figure 1: Gene expression analysis. (a) The level of relative ANGPTL4 mRNA expression in HUVECs from healthy (control) and GDM patients. (b) The level of relative ADIPOQ mRNA expression in multipotent mesenchymal stromal cells (MSC) (as negative control), adipocytes (as positive control), and HUVECs. (c) The level of relative LEPR mRNA expression in HUVECs from healthy (control) and GDM patients. (d) Correlation between the relative LEPR mRNA expression in HUVECs and the level of leptin in the cord blood.

it is due to the fact that the difference in target glycaemic levels is not significant enough to affect the expression level of ANGPTL4, at least on such a small sample. Another plausible explanation is that the reduced level of activity of ANGPTL4 is transmitted at the genetic level to newborns from their mothers. Maybe, the reduced level of activity of ANGPTL4 contributes to the development of GDM in the mothers, that is, it is the cause, not the consequence of hyperglycaemia.

This hypothesis is supported by the data of $\mathrm{Xu}$ et al. on the lower level of ANGPTL4 in patients with type 2 diabetes whose pathogenesis is close to GDM [9]. However, this hypothesis is contradicted by the data of Ortega-Senovilla et al., indicating that maternal serum ANGPTL4 concentrations showed no difference between the control and GDM women [13].

Moreover, opposite to our data, Ortega-Senovilla et al. showed that serum ANGPTL4 concentrations in cord serum were higher in those from GDM than those from control pregnancies [13]. We found no difference in the level of ANGPTL4 in the cord serum. There seems to be no correlation between the levels of ANGPTL4 protein in the cord serum and ANGPTL4 gene expression in one of the fetal tissues (HUVEC). It is plausible that the main source of cord serum ANGPTL4 is some other fetal tissue (e.g., the liver). The functional consequences of the downregulation of ANGPTL4 mRNA levels in HUVECs in GDM remain to be identified.

In addition, other factors besides intrauterine hyperglycaemia may affect the activity of a number of genes, including ANGPTL4, in the fetus. Known factors that affect the weight of the newborn are the body mass index (BMI) of the mother and maternal gestational weight gain. Obviously, these parameters are influenced by the mother's lifestyle (the quantitative and qualitative composition of the diet and the level of physical activity). 
It is known that ANGPTL4 can be regulated by diet $[38,39]$. The diet interventions leading to NEFA increase in the blood (high-fat diet, a very low-energy diet, and fasting) were shown to upregulate the plasma level of ANGPTL4 [38].

This upregulation has been confirmed likewise in vitro as the expression of ANGPTL4 is upregulated in response to exposure to fatty acids in cell studies [40].

Our study established a significantly lower pregnancy weight gain in GDM patients compared to controls which is obviously due to diet adherence by patients.

Further studies are needed to clarify the cause-and-effect relationship between GDM and the level of expression of ANGPTL4 gene in HUVEC.

The level of C-peptide in the cord blood is commonly used as a marker of fetal hyperinsulinemia [41]. The data presented by HAPO study has shown associations between increasing levels of fasting, 1-hour, and 2-hour plasma glucose obtained on oral glucose-tolerance testing and cord blood serum C-peptide level above the 90th percentile [41]. We did not reveal any difference in the level of C-peptide among the groups. It could be a result of the treatment that was efficient to reduce fetal hyperinsulinemia in both GDM groups or it may be due to a small sample size.

The strength of our study is the design of the RCT of different glycaemic targets for women with GDM which allows at testing cause-and-effect relationships. The weakness of the study, besides its relatively small sample size, is the lack of information about maternal levels of the studied gene expression.

\section{Conclusion}

Our study established positive association of cord leptin levels and LAR with target levels of glycaemia during pregnancy in women with GDM. Further investigation into long-term consequences of cord leptin concentrations is required.

We also found a decrease in the expression of ANGPTL4 in HUVECs of neonates from mothers with GDM. However, we could not prove the causal relationship between intrauterine hyperglycaemia and the expression of the ANGPTL4 gene, given the absence of differences between the level of expression of ANGPTL4 in groups with different glycaemic targets. This relationship remains to be clarified.

\section{Data Availability}

The data used to support the findings of this study are available from the corresponding author upon request.

\section{Ethical Approval}

The authors assert that all procedures contributing to this work comply with the ethical standards of the relevant national guidelines on human experimentation and with the Helsinki Declaration of 1975, as revised in 2008, and have been approved by the institutional committee of ANMRC (Protocol 119).

\section{Conflicts of Interest}

The authors declare no conflict of interest.

\section{Acknowledgments}

The study was funded by the Russian Science Foundation (Project no. 17-75-30052).

\section{References}

[1] D. Dabelea, "The predisposition to obesity and diabetes in offspring of diabetic mothers," Diabetes Care, vol. 30, Supplement 2, pp. S169-S174, 2007.

[2] N. El Hajj, E. Schneider, H. Lehnen, and T. Haaf, "Epigenetics and life-long consequences of an adverse nutritional and diabetic intrauterine environment," Reproduction, vol. 148, no. 6, pp. R111-R120, 2014.

[3] T. J. Kieffer and J. F. Habener, "The adipoinsular axis: effects of leptin on pancreatic $\beta$-cells," American Journal of Physiology Endocrinology and Metabolism, vol. 278, no. 1, pp. E1-E14, 2000.

[4] M. Blüher and C. S. Mantzoros, "From leptin to other adipokines in health and disease: facts and expectations at the beginning of the 21st century," Metabolism Clinical and Experimental, vol. 64, no. 1, pp. 131-145, 2015.

[5] A. M. Teague, D. A. Fields, C. E. Aston, K. R. Short, T. J. Lyons, and S. D. Chernau, "Cord blood adipokines, neonatal anthropometrics and postnatal growth in offspring of Hispanic and Native American women with diabetes mellitus," Reproductive Biology and Endocrinology, vol. 13, no. 1, p. 68, 2015.

[6] I. Y. Kon, N. M. Shilina, M. V. Gmoshinskaya, and T. A. Ivanushkina, "The study of breast milk IGF-1, leptin, ghrelin and adiponectin levels as possible reasons of high weight gain in breast-fed infants," Annals of Nutrition \& Metabolism, vol. 65, no. 4, pp. 317-323, 2014.

[7] C. S. Mantzoros, S. L. Rifas-Shiman, C. J. Williams, J. L. Fargnoli, T. Kelesidis, and M. W. Gillman, "Cord blood leptin and adiponectin as predictors of adiposity in children at 3 years of age: a prospective cohort study," Pediatrics, vol. 123, no. 2, pp. 682-689, 2009.

[8] H. K. Kim, B. S. Youn, M. S. Shin et al., "Hypothalamic Angptl4/Fiaf is a novel regulator of food intake and body weight," Diabetes, vol. 59, no. 11, pp. 2772-2780, 2010.

[9] A. Xu, M. C. Lam, K. W. Chan et al., "Angiopoietin-like protein 4 decreases blood glucose and improves glucose tolerance but induces hyperlipidemia and hepatic steatosis in mice," Proceedings of the National Academy of Sciences of the United States of America, vol. 102, no. 17, pp. 6086-6091, 2005.

[10] A. Köster, Y. B. Chao, M. Mosior et al., "Transgenic angiopoietin-like (Angptl)4 overexpression and targeted disruption of Angptl4 and Angptl3: regulation of triglyceride metabolism," Endocrinology, vol. 146, no. 11, pp. 4943-4950, 2005.

[11] M. R. J. N. Robciuc, A. Ortega-Alonso, H. Tyynismaa et al., "Serum angiopoietin-like 4 protein levels and expression in adipose tissue are inversely correlated with obesity in monozygotic twins," Journal of Lipid Research, vol. 52, no. 8, pp. 15751582, 2011.

[12] L. Pirc, J. Owens, C. Crowther, K. Willson, M. De Blasio, and J. Robinson, "Mild gestational diabetes in pregnancy and the adipoinsular axis in babies born to mothers in the ACHOIS randomised controlled trial," BMC Pediatrics, vol. 7, no. 1, p. $18,2007$. 
[13] H. Ortega-Senovilla, U. Schaefer-Graf, K. Meitzner, M. Abou-Dakn, and E. Herrera, "Decreased concentrations of the lipoprotein lipase inhibitor angiopoietin-like protein 4 and increased serum triacylglycerol are associated with increased neonatal fat mass in pregnant women with gestational diabetes mellitus," The Journal of Clinical Endocrinology and Metabolism, vol. 98, no. 8, pp. 3430-3437, 2013.

[14] M. Meller, C. Qiu, S. Vadachkoria, D. F. Abetew, D. A. Luthy, and M. A. Williams, "Changes in placental adipocytokine gene expression associated with gestational diabetes mellitus," Physiological Research, vol. 55, no. 5, pp. 501-512, 2006.

[15] P. Di Fulvio, A. Pandolfi, G. Formoso et al., "Features of endothelial dysfunction in umbilical cord vessels of women with gestational diabetes," Nutrition, Metabolism \& Cardiovascular Diseases, vol. 24, no. 12, pp. 1337-1345, 2014.

[16] I. I. Dedov, V. I. Krasnopol'skiy, and G. T. Sukhikh, "Russian National Consensus Statement on gestational diabetes: diagnostics, treatment and postnatal care," Diabetes mellitus, vol. 15, no. 4, pp. 4-10, 2012.

[17] B. E. Metzger, "International Association of Diabetes and Pregnancy Study Groups recommendations on the diagnosis and classification of hyperglycemia in pregnancy," Diabetes Care, vol. 33, no. 3, pp. 676-682, 2010.

[18] E. Pustozerov, P. Popova, A. Tkachuk, Y. Bolotko, Z. Yuldashev, and E. Grineva, "Development and evaluation of a mobile personalized blood glucose prediction system for patients with gestational diabetes mellitus," JMIR Mhealth Uhealth, vol. 6, no. 1, article e6, 2018.

[19] E. A. Jaffe, R. L. Nachman, C. G. Becker, and C. R. Minick, "Culture of human endothelial cells derived from umbilical veins. Identification by morphologic and immunologic criteria," The Journal of Clinical Investigation, vol. 52, no. 11, pp. 2745-2756, 1973.

[20] M. Puzanov, L. Vasilyeva, P. Popova, E. N. Grineva, and R. I. Dmitrieva, "New approach to cryopreservation of primary noncultivated human umbilical vein endothelium in biobanking," Biopreservation and Biobanking, vol. 16, no. 2, pp. 114119, 2018.

[21] K. J. Livak and T. D. Schmittgen, "Analysis of relative gene expression data using real-time quantitative PCR and the 2(-delta delta $\mathrm{C}(\mathrm{T})$ ) method," Methods, vol. 25, no. 4, pp. 402-408, 2001.

[22] R. I. Dmitrieva, I. R. Minullina, A. A. Bilibina, O. V. Tarasova, S. V. Anisimov, and A. Y. Zaritskey, "Bone marrow- and subcutaneous adipose tissue-derived mesenchymal stem cells: differences and similarities," Cell Cycle, vol. 11, no. 2, pp. 377-383, 2012.

[23] P. Popova, K. Castorino, E. Grineva, and D. Kerr, "Gestational diabetes mellitus diagnosis and treatment goals: measurement and measures," Minerva Endocrinologica, vol. 41, pp. 421-432, 2016.

[24] I. Blumer, E. Hadar, D. R. Hadden et al., "Diabetes and pregnancy: an endocrine society clinical practice guideline," The Journal of Clinical Endocrinology and Metabolism, vol. 98, no. 11, pp. 4227-4249, 2013.

[25] Canadian Diabetes Association Clinical Practice Guidelines Expert Committee, D. Thompson, H. Berger et al., "Diabetes and pregnancy," Canadian Journal of Diabetes, vol. 37, pp. S168-S183, 2013.

[26] American Diabetes Association, "Standards of medical care in diabetes-2017,” Diabetes Care, vol. 40, pp. S1-S135, 2017.
[27] B. E. Metzger, T. A. Buchanan, D. R. Coustan et al., "Summary and recommendations of the Fifth International WorkshopConference on Gestational Diabetes Mellitus," Diabetes Care, vol. 30, Supplement 2, pp. S251-S260, 2007.

[28] M. B. Landon, C. Y. Spong, E. Thom et al., "A multicenter, randomized trial of treatment for mild gestational diabetes," The New England Journal of Medicine, vol. 361, no. 14, pp. 1339-1348, 2009.

[29] R. Martis, J. Brown, J. Alsweiler, T. J. Crawford, and C. A. Crowther, "Different intensities of glycaemic control for women with gestational diabetes mellitus," Cochrane Database of Systematic Reviews, no. 4, article CD011624, 2016.

[30] T. Parsons, C. Power, and O. Manor, "Fetal and early life growth and body mass index from birth to early adulthood in 1958 British cohort: longitudinal study," BMJ, vol. 323, no. 7325, pp. 1331-1335, 2001.

[31] K. K. Ong, M. L. Ahmed, A. Sherriff et al., "Cord blood leptin is associated with size at birth and predicts infancy weight gain in humans," The Journal of Clinical Endocrinology \& Metabolism, vol. 84, no. 3, pp. 1145-1148, 1999.

[32] M. Parker, S. L. Rifas-Shiman, M. B. Belfort et al., "Gestational glucose tolerance and cord blood leptin levels predict slower weight gain in early infancy," The Journal of Pediatrics, vol. 158, no. 2, pp. 227-233, 2011.

[33] C. N. Chaoimh, D. M. Murray, L. C. Kenny, A. D. Irvine, J. O. Hourihane, and M. Kiely, "Cord blood leptin and gains in body weight and fat mass during infancy," European Journal of Endocrinology, vol. 175, no. 5, pp. 403-410, 2016.

[34] M. Ballesteros, I. Simón, J. Vendrell et al., "Maternal and cord blood adiponectin multimeric forms in gestational diabetes mellitus: a prospective analysis," Diabetes Care, vol. 34, no. 11, pp. 2418-2423, 2011.

[35] Z.-C. Luo, A.-M. Nuyt, E. Delvin et al., "Maternal and fetal leptin, adiponectin levels and associations with fetal insulin sensitivity," Obesity, vol. 21, no. 1, pp. 210-216, 2013.

[36] J. Chen, B. Tan, E. Karteris et al., "Secretion of adiponectin by human placenta: differential modulation of adiponectin and its receptors by cytokines," Diabetologia, vol. 49, no. 6, pp. 1292 1302, 2006.

[37] J. E. Caminos, R. Nogueiras, R. Gallego et al., "Expression and regulation of adiponectin and receptor in human and rat placenta," The Journal of Clinical Endocrinology \& Metabolism, vol. 90, no. 7, pp. 4276-4286, 2005.

[38] J. T. Jonker, J. W. Smit, S. Hammer et al., "Dietary modulation of plasma angiopoietin-like protein 4 concentrations in healthy volunteers and in patients with type 2 diabetes," The American Journal of Clinical Nutrition, vol. 97, no. 2, pp. 255-260, 2013.

[39] S. Kersten, L. Lichtenstein, E. Steenbergen et al., "Caloric restriction and exercise increase plasma ANGPTL4 levels in humans via elevated free fatty acids," Arteriosclerosis, Thrombosis, and Vascular Biology, vol. 29, no. 6, pp. 969-974, 2009.

[40] P. Gonzalez-Muniesa, C. de Oliveira, F. Perez de Heredia, M. P. Thompson, and P. Trayhurn, "Fatty acids and hypoxia stimulate the expression and secretion of the adipokine ANGPTL4 (angiopoietin-like protein 4/ fasting-induced adipose factor) by human adipocytes," Journal of Nutrigenetics and Nutrigenomics, vol. 4, no. 3, pp. 146-153, 2011.

[41] HAPO Study Cooperative Research Group, B. E. Metzger, L. P. Lowe et al., "Hyperglycemia and adverse pregnancy outcomes," The New England Journal of Medicine, vol. 358, pp. 1991-2002, 2008. 


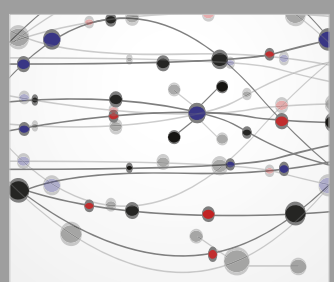

The Scientific World Journal
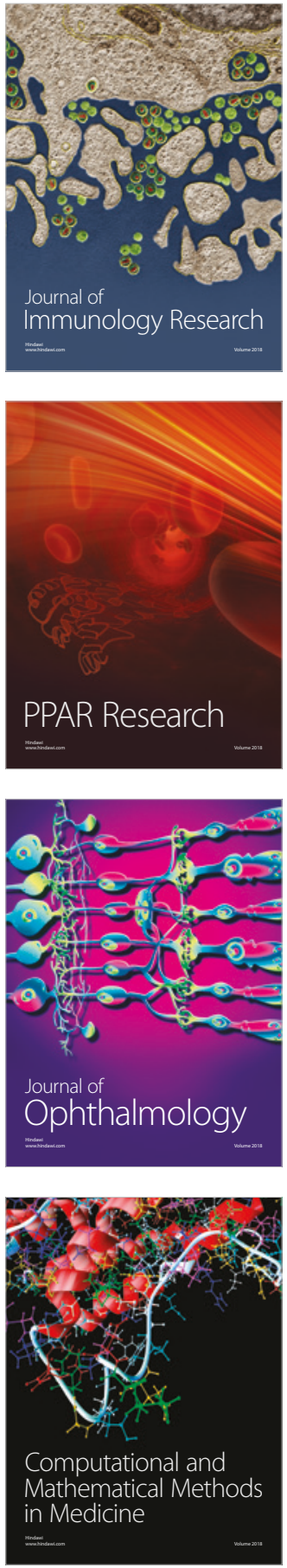

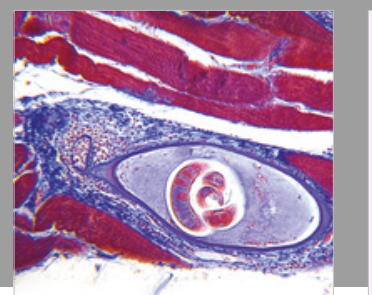

Gastroenterology Research and Practice

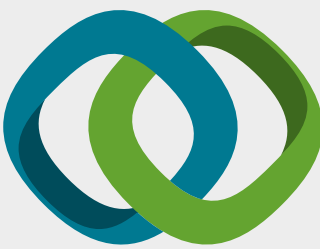

\section{Hindawi}

Submit your manuscripts at

www.hindawi.com
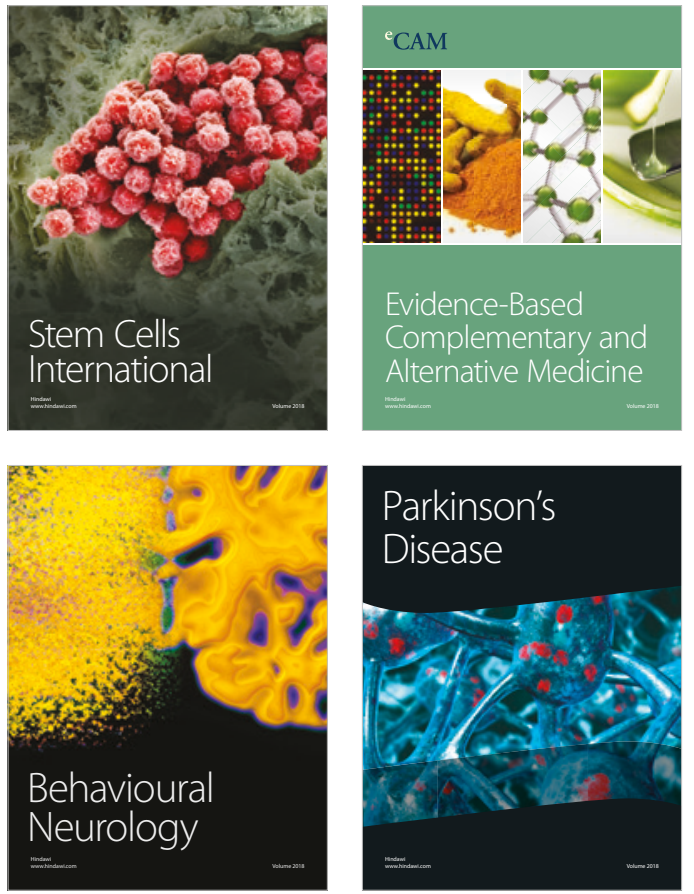

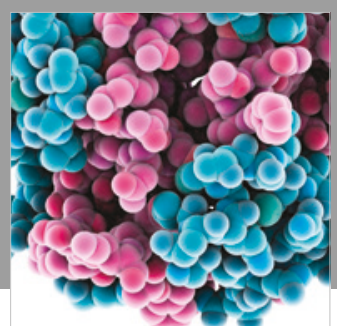

ournal of

Diabetes Research

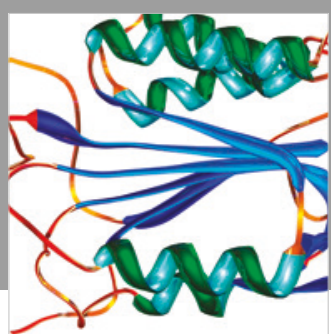

Disease Markers
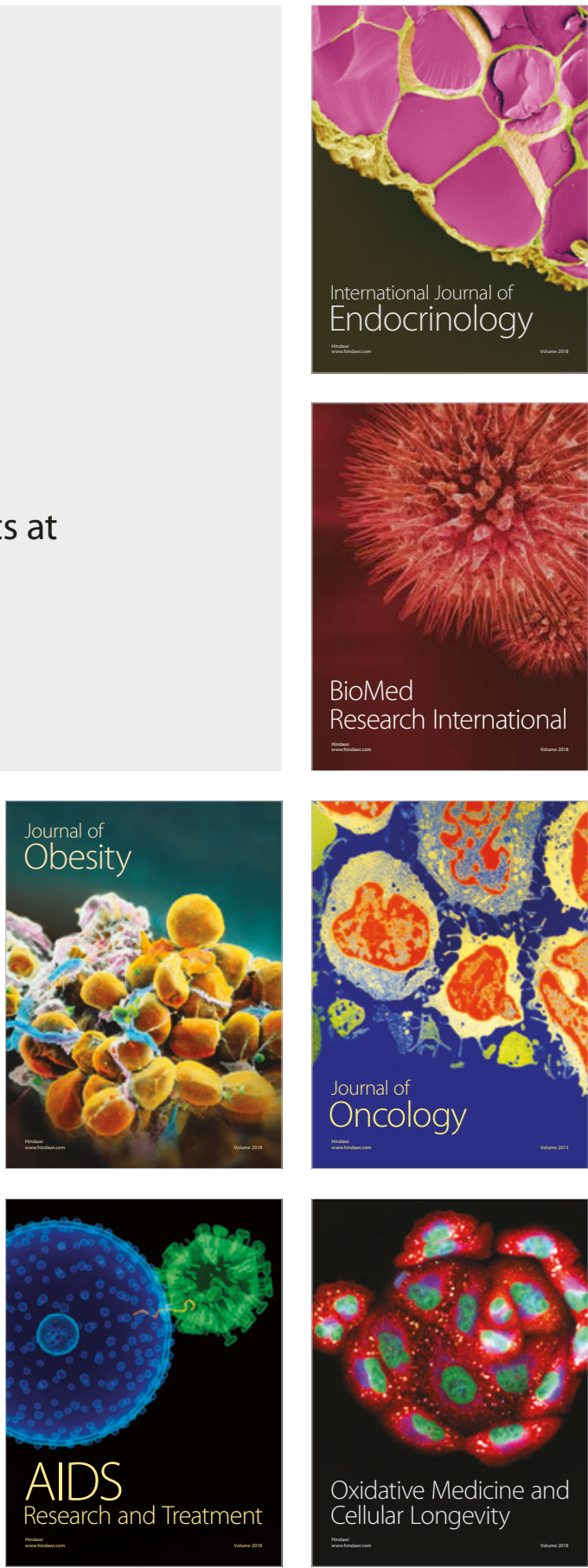